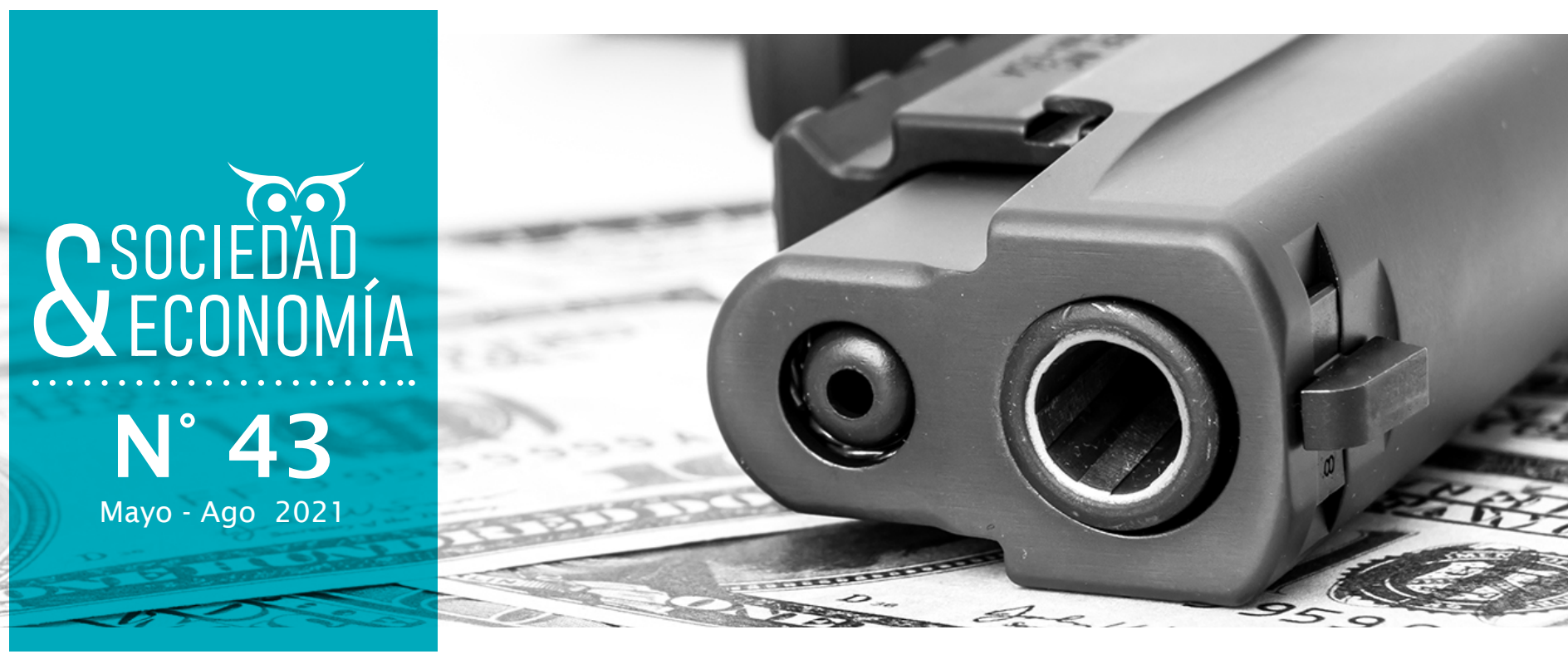

\title{
Incidencia multidimensional de la oferta criminal de Colombia desde un enfoque de ecuaciones simultáneas
}

Multidimensional Incidence of Colombia's Criminal Offer from a Simultaneous Equations Approach

Hector Daniel Martínez-Duarte ${ }^{1}$

Alcaldía de San José de Cúcuta, Cúcuta, Colombia.

ㅅ․․martinezd@uniandes.edu.co

(D) https://orcid.org/0000-0002-4612-6974

Angel David Ramírez-Romero ${ }^{2}$

Alcaldía de San José de Cúcuta, Cúcuta, Colombia.

(2) ad.ramirezr12@uniandes.edu.co

(D) https://orcid.org/0000-0002-1259-2870

\section{Eder Alexander Botello-Sánchez ${ }^{3}$}

Universidad de Pamplona, Villa del Rosario, Colombia.

@alexander.botello@unipamplona.edu.co

(1) https://orcid.org/0000-0003-4363-5551

Recibido: 21-05-2020

Aceptado: 08-12-2020

Publicado: 01-05-2021

Economista.

2 Economista.

3 Magíster en estudios y gestión del desarrollo. 
Sociedad y Economía N`43 (Mayo - Ago 2021) / e-ISSN: 2389-9050 / e1029738

Hector Daniel Martínez-Duarte, Angel David Ramírez-Romero y Eder Alexander Botello-Sánchez

\title{
Resumen
}

El crimen ha sido explicado a partir de distintos enfoques y disciplinas. Esta investigación se traza, desde un enfoque económico, la determinación del grado de incidencia de los factores sociodemográficos, económicos, institucionales y geográficos en la oferta criminal de Colombia y sus departamentos durante el periodo 2010 a 2017; para ello, se hace uso del método cuantitativo de ecuaciones simultáneas, y bajo la estimación de Máxima Verosimilitud con Información Completa, se pudieron obtener como principales hallazgos: 1) el crimen presenta relaciones significativas con las variables sociodemográficas, económicas, institucionales y geográficas. 2) El número de agentes policiales de un departamento administrativo depende positivamente del número de crímenes reportados y el tamaño del territorio. Finalmente, se puede concluir que la intervención estatal direccionada a la mitigación del crimen debe considerar las relaciones interdisciplinarias que interactúan en torno al fenómeno para que las políticas públicas sean eficientes.

Palabras clave: ecuaciones simultáneas; territorio; crimen; seguridad del Estado.

Clasificación JEL: H56; C24; C3; K42.

\begin{abstract}
Crime has been explained from different approaches and disciplines. From an economic approach, this investigation traces the determination of the degree of incidence of sociodemographic, economic, institutional, and geographic factors, in the criminal offer of Colombia and its departments during the period 2010 to 2017; for this, the quantitative method of simultaneous equations is used, also, under the modification of Maximum Likelihood with Complete Information, the following main findings are obtained: 1) crime presents relationships related to sociodemographic, economic, institutional and geographic variables. 2) The number of police officers of an administrative department positively dependent on the number of crimes reported and the size of the territory. Finally, it can be concluded that state intervention aimed at crime mitigation must consider interdisciplinary relationships that interact around the phenomenon so that public policies are efficient.
\end{abstract}

Keywords: Simultaneous equations; territory; crime; state security.

JEL classification: H56; C24; C3; K42.

\section{Finaciación}

Este trabajo es resultado de un proyecto de aula que posteriormente le otorgaría a Héctor Daniel Martínez el título de economista. No tuvo financiación.

\section{Conflicto de interés}

Los autores declaran no tener ningún conflicto de interés en la publicación de este artículo.

\section{(ल) $(\Theta)$ Este trabajo está bajo la licencia Atribución-No-Comercial 4.0 Internacional}

\section{¿Cómo citar este artículo?}

Martínez-Duarte, H. D., Ramírez-Romero, A. D. y Botello-Sánchez, E. A. (2021). Incidencia multidimensional de la oferta criminal de Colombia desde un enfoque de ecuaciones simultáneas. Sociedad y economía, (43), e1 029738. https://doi.org/10.25100/sye.v0i43.9738 


\section{Introducción}

El crimen, como lo expone Beccaria (1828), es un fenómeno que ha estado presente desde que la humanidad decidió organizarse para vivir en comunidad. Desde entonces, sus matices han variado conforme avanzan las sociedades, al punto que hoy en día, la lucha contra este se ha vuelto una política crucial de los Estados modernos (Chabat, 2010). Por otra parte, las disciplinas e hipótesis que históricamente se han encargado de abordar el crimen también han cambiado. Tan es así que la violencia y el crimen son considerados fenómenos multicausales y, por ende, su conceptualización está lejos de ser universalmente aceptada o consensuada. A raíz de lo anterior, no han sido escasos los acercamientos de las diciplinas entre sí, con el ánimo de ofrecer una comprensión mucho más amplia, cuyo espectro académico se concierne multidimensional e interdisciplinario.

Colombia, desde la época de la conquista hasta finales del siglo XX, se ha caracterizado por tener múltiples eventos de violencia. El reporte de Verisk (BBC, 2016), situó al país en el podio de criminalidad y disuasión a nivel internacional, ocupando el puesto 12 entre los países con mayor índice de criminalidad a nivel mundial. El crimen se entiende como homicidios, hurtos, delitos sexuales, amenazas y terrorismo; hechos que han sido una constante histórica y un flagelo del estado social de derecho de los colombianos que aún queda por superar (Robinson y Urrutia, 2007).

Ahora bien, el crimen recubre prácticamente todo el territorio colombiano y, por ende, cada una de sus regiones requiere ser analizada detalladamente. La escasa literatura que aborda este tema desde un enfoque de ecuaciones simultáneas brinda la oportunidad para dar una explicación frente al fenómeno de estudio. Sumado a esto, la coyuntura que atraviesa el país y sus regiones en torno al proceso de finalización del conflicto armado hace pertinente la presente investigación, para la indagación, exploración y análisis del crimen.

Teniendo en cuenta lo anterior, el presente artículo se traza como objetivo determinar la incidencia de los factores económicos, sociodemográficos, institucionales y geográficos en la oferta criminal de Colombia en el periodo 2010 -2017. En ese orden de ideas, los investigadores plantean una hipótesis que sostiene que no todos los factores inciden de igual forma o al unísono en la oferta criminal del país, sino que al interior de cada factor existen variables promotoras y disuasorias de la oferta criminal de Colombia en el periodo de estudio.

La contemplación de múltiples factores y su incidencia en el crimen obedece a la revisión de la literatura, en la que se observa el paso por diversas disciplinas. Una primera línea se orienta a un enfoque contractualista, que parte de los acuerdos entre los seres humanos y las normas acordadas en sociedad. Un primer acercamiento a la discusión en torno al crimen se remonta a la ilustración, cuando Marchese Di Beccaria (1828) indagaba sobre el fenómeno en De los delitos y las penas; luego acompañado por Bentham (1789), quien teorizó el delito como una decisión (de muchas que los individuos eligen). Más adelante, como bien expone Anzola et al. (2005), el crimen era un asunto considerado propio o exclusivo de la psicología y la sociología.

No obstante, con el tiempo, la economía, la demografía e incluso la historia, dieron apertura a la formación de variados grupos de estudio e investigación frente al fenómeno, robusteciendo la escuela positivista de la criminología. Por otra parte, el enfoque neoclásico es el de mayor interés para la presente investigación, toda vez que posiciona la economía como una de las líneas más fuertes. Desde la publicación de Becker (1968), pasando por Ramírez-de-Garay (2014), se logró relacionar el ciclo y la privación económica como factores que inciden en la manifestación del crimen, desde una óptica psíquica, individual y determinista.

Adicionalmente, como se mencionó con anterioridad, otras disciplinas hicieron lo propio. Ramirez-de-Garay (2014) expone cómo el crimen se relaciona con factores sociales; otros, como Bandrés y Díez-Ticio (2001), argumentan que este se debe simultáneamente a cuestiones normativas, estructurales y contextuales, 
Sociedad y Economía N 43 (Mayo - Ago 2021) / e-ISSN: 2389-9050 / e1029738

Hector Daniel Martínez-Duarte, Angel David Ramírez-Romero y Eder Alexander Botello-Sánchez

es decir, el crimen es un fenómeno multicausal. Para corroborar lo anterior, en esta investigación se utiliza el método cuantitativo de ecuaciones simultáneas, mediante la estimación de Máxima Verosimilitud con Información Completa (MVIC). En ella se plantea un modelo matemático de la oferta criminal que se fundamenta en la literatura consultada y la información disponible, en donde fueron valiosos los aportes de Becker (1968), Núñez et al. (2003), Rodríguez (2003), Bandrés y Díez-Ticio (2001); Gómez et al. (2020), y Martínez (2001). A partir de esto, se construye un sistema de ecuaciones producto de la unión de la oferta del crimen, la eficiencia y dotación policial.

Las metodologías usadas para abordar el crimen y los factores que inciden en este son diversos. Se tiene desde la línea económica, análisis macro analíticos de modelos regresivos como los de Bandrés y Díez-Ticio (2001), como también se encuentran análisis micro analíticos en los que se construye una función de presión descriptiva Buonanno (2003). Por su parte, otros estudios como los de Galindo y Catalán (2007), y Martínez (2001), utilizaron estimaciones paramétricas, en las que se incluyeron variables institucionales, culturales y sociales. Sumado a esto, otras metodologías como las de modelos paramétricos de panel - estático, utilizados por Núñez et al. (2003), incorporan adicionalmente la variable geográfica, al igual que Gómez y Serrano (2015) en México, quien estudia la geografía criminal mediante la econometría espacial. Para el caso colombiano, se tiene un experimento de campo reciente por parte de Collazos et al. (2020), donde muestra cómo la vigilancia en puntos calientes de la ciudad de Medellín mejora la percepción de seguridad en el corto plazo y reducción del robo de vehículos, pero no tiene efectos en otros tipos de crimen. Por otro lado, se tienen los estudios de Mancera (2010), Carranza-Romero et al. (2011), entre otros, los cuales utilizaron desde análisis de series de tiempo hasta métodos econométricos de ecuaciones simultáneas como el implementado por la presente investigación.

Como podrá observar el lector, la literatura ofrece una amplia diversidad tanto de enfo- ques como de metodologías para el abordaje de la oferta criminal. No obstante, se observa un lugar común: la mayoría recurre a la inferencia estadística, mediante el uso del micro y macro análisis, en donde se expone que el crimen es un fenómeno que implica costos sociales que impactan negativamente en el desarrollo y bienestar de una sociedad. Asimismo, este fenómeno puede ser mitigado a través de la interacción de los factores sociodemográficos, económicos, geográficos e institucionales que convergen en un territorio.

Finalmente, el artículo se estructura en cinco apartados: el primero es la presente introducción; el segundo, el desarrollo teórico en el que se expone la adopción del referente teórico; el tercero, los detalles de la metodología aplicada; en el cuarto apartado, se presentan los resultados obtenidos y, por último, las conclusiones derivadas de la investigación.

\section{Enfoque teórico}

El crimen es un fenómeno que ha existido desde los orígenes de las sociedades hasta el siglo XXI. Este se define a partir de la conformación de las leyes que permitieron la creación de los Estados bajo la teoría contractualista. Diversos autores han abordado esta temática desde inicios del siglo XIX, lo cual produjo que desde variados enfoques se identificara de mejor manera sus causas, comportamiento y consecuencias.

Anzola et al. (2005) expone que, desde los inicios, la psicología marcó una pauta en la forma de entender el delito, ya no como una expresión despreciable de la humanidad, sino como una de las actividades cotidianas de los seres humanos, a la cual las autoridades debían hacerle frente. También se pudo observar un aporte de la sociología en cuanto a la caracterización del perfil del reo, como, por ejemplo, su educación, edad, género, etc. Según Estepa (2005), lo anterior permitió que "(...) dentro de ciencias como la historia, la demografía y la economía, se inicie la investigación del crimen desde dos líneas paralelas de investigación" (p. 299).

La primera de estas líneas se dio en Francia por parte de la escuela de los Annales que se inte- 
resó por la totalidad de las actividades humanas, dedicándose al monitoreo cuantitativo de los procesos de judicialización -desde la captura hasta la condena- en determinado espacio geográfico. Simultáneamente, la segunda línea emergió en Inglaterra, y su característica principal fue el estudio del crimen desde un enfoque marxista, el cual se daba a entender como un acto de opresión y el uso de las leyes como herramienta de la clase dominante. Estas divisiones serán eclipsadas por la corriente económica predominante durante los últimos años. Los modelos matemáticos serían la base fundamental para la economía al momento de explicar un fenómeno social, que años atrás tenía meramente implicaciones éticas y filosóficas, para así, eventualmente, robustecer la escuela positivista de criminología.

Siegel (2011) define la Criminología como aquella disciplina que emplea el método científico para estudiar la naturaleza, extensión, causas y control de la conducta criminal. En donde destaca la estrecha relación interdisciplinar que conglomera al estudio del crimen con un fin común, el cual es analizar y mitigar sus consecuencias. Es a partir de los antecedentes mencionados que el interés en el estudio del crimen se hace tangible. Dado el contexto y la trayectoria del fenómeno, se han creado organizaciones como The History of Crime and Criminal Justice y grupos de investigación para el tema, así como investigaciones y aportes metodológicos para abordar una problemática que continuaría incrementándose con el desarrollo de los países industrializados durante el siglo XX.

Hasta fines de la década del 60 la literatura sobre crimen se encontraba dominada por psicólogos y sociólogos que consideraban que la criminalidad estaba determinada por factores como educación, entorno social, herencia, nivel de pobreza, desocupación, etc. La conclusión de política económica a que llegaba esta corriente era que no debían destinarse recursos a la seguridad ya que no tenían ninguna influencia sobre el crimen (Balbo y Posadas, 1998, p. 1).

Para ese entonces, así como la economía podía explicar el comportamiento de indi- viduos en el mercado, Gary Becker (1968) quiso explicar el comportamiento del delincuente basando su teoría en dos autores del siglo XVIII, con los cuales compartió el interés desde el punto de vista económico por el crimen. Los economistas clásicos y próceres de la ilustración tales como Cesare Beccaria (1 828) y Jeremy Bentham (1789) también dedicaron una atención devota a la explicación del crimen en términos económicos racionales y a la formulación de las reglas óptimas para castigar a los delincuentes, basadas en principios de utilidad.

Durante la ilustración, muchos pensadores como Montesquieu (1989) habían tocado estos temas, pero sin darles un aporte estructurado a la creencia de separar el pecado del delito. Como el delito se consideró un asunto meramente humano, Bentham (1789) fue de los primeros que teorizó el delito como una decisión. Así mismo, Beccaria (1828) lo consideró como un fenómeno inherente al desarrollo de las sociedades. Como se mencionó al principio, hacia finales del siglo XIX este vínculo entre la economía y el crimen fue tomando interés en las nacientes ciencias sociales. Según Ramírez-de-Garay (2014):

La escuela positivista de criminología adoptó rápidamente los preceptos de la economía política clásica para trabajar con su objeto de estudio. Dicha idea incluía una definición amplia de la economía como: las interacciones económicas entre los individuos y el Estado y los correspondientes procesos de producción y distribución de bienes económicos. Esta aproximación clásica se convirtió rápidamente en la base conceptual de dos explicaciones posteriores sobre la relación economía-crimen: el ciclo económico, y la privación económica (p. 264).

La primera de estas corrientes consistió en explicar el crimen como una respuesta al ciclo económico, es decir, como una relación inversamente proporcional del auge económico y las tasas de criminalidad. Sin embargo ante las nuevas metodologías, estos argumentos quedaban cada vez más sin soporte empírico. Por otro lado, la privación económica postulaba que la actividad econó- 
Sociedad y Economía N` 43 (Mayo - Ago 2021) / e-ISSN: 2389-9050 / e1029738

Hector Daniel Martínez-Duarte, Angel David Ramírez-Romero y Eder Alexander Botello-Sánchez

mica generaba una desigualdad de oportunidades que desembocaban en una criminalidad mayor por parte de esos segmentos de la población. De acuerdo con lo anterior, se puede esperar que no solo con el auge económico las tasas de criminalidad aumenten, sino también, los tipos de delitos; ejemplo de ello, pueden ser los delitos informáticos. De esta manera, se observa que la evolución de este fenómeno está unida, como se teorizó anteriormente, a los campos donde la humanidad hace un avance.

Así las cosas, para finales del siglo XX la discusión gestó los principios y bases del enfoque teórico con respecto al nexo entre la actividad criminal y las cuestiones propias de la economía; situación en donde el enfoque clásico de la economía política, que hasta el momento abordaba el fenómeno, fue sustituido paulatinamente por la escuela neoclásica de la economía, y con ello surgió una nueva forma de explicar y abordar la relación.

Según Ramírez-de-Garay (2014), el enfoque neoclásico arribó con tanta fuerza que prácticamente reemplazó todos los conceptos derivados de la economía política. Balbo y Posadas (1998) afirman que:

La publicación del trabajo de Gary Becker (1968) cambia por completo la óptica del problema y se convierte en el referente teórico de la literatura de crimen y castigo. Considera al crimen como una actividad económica más dentro de la economía $\mathrm{y}$ al criminal como un individuo racional. Becker basa el análisis en la teoría del bienestar, y en cierto sentido es una versión primitiva del problema del principal agente, donde la sociedad es el principal y los criminales son los agentes. Supone que el criminal comete un delito solo si la utilidad esperada de una actividad ilegal excede a la utilidad que obtendría si emplea ese tiempo y esos recursos en una actividad legal (p. 2).

La esencia del tema descansa en el supuesto según el cual los actores que delinquen actúan de manera racional, y por tal razón responden a incentivos, positivos o negati- vos. Dado lo anterior, la decisión microanalítica de delinquir se reduce a un problema de maximización de utilidad.

La teoría neoclásica también afirma que el volumen de delincuentes efectivos ${ }^{4}$ en la población depende de la dotación de recursos y el grado de institucionalidad. Esto es análogo a lo que Ehrlich (1996) comenta sobre Adam Smith, donde este último observó que tanto el crimen como la demanda de protección contra la actividad delictiva estaban motivados por la acumulación de propiedad. Así las cosas, Gary Becker et al. (1990) plasma en su obra El Enfoque Económico del Comportamiento Humano que el individuo (o ser racional social) toma en cuenta las ganancias o pérdidas de las consecuencias de su conducta.

Becker et al. (1990) estudia el comportamiento humano con base en los incentivos o preferencias que se desglosan de las acciones de estos. En consecuencia, el planteamiento Beckeriano refleja un estudio del crimen marcado tanto por un enfoque psíquico e individual, como también por uno determinista que se encarga de englobar las transformaciones sociales.

Aunque algunos factores pueden no ser determinantes para definir las causas delictivas, tales como las condiciones relacionadas con el género, sí que son una buena herramienta para tomar decisiones y distribuir de forma adecuada los ingresos que se destinan al combate del crimen y la mitigación de sus efectos y costos sociales, especialmente en países conflictivos cuyos índices de criminalidad son elevados.

Finalmente, las aproximaciones desde distintas disciplinas han dado lugar a discusiones muy enriquecedoras. Sin embargo, autores como Drake y Simper (2005), y Garoupa (2003), argumentan que la teoría clásica de la economía sigue siendo la mejor aproxima-

4 Entiéndase como los agentes económicos que se dedican a la actividad criminal. 
ción para lograr la aplicación de la ley. Como resultado se obtiene un grupo heterogéneo de explicaciones $y$, aunque se han logrado amplios avances en el estudio de la criminalidad, aún hacen falta estudios más amplios sobre sus causas, como lo menciona Estepa (2005):

(...) las causas y la incidencia del crimen no pueden explicarse atendiendo a la influencia de un único factor, sino que dependen de la conjunción de los efectos de un amplio conjunto de variables económicas, sociales, políticas, institucionales, culturales, mentales, etc., de ahí que la explicación de los cambios en las tasas de delincuencia y en la relación interna de las categorías delictivas pase por la formulación de teorías globalizantes (p. 305).

Asimismo, de acuerdo al planteamiento de Ramirez-de-Garay (2014), las explicaciones criminales se ven altamente correlacionadas con los factores sociales que crean el escenario para que se finiquiten los actos. Por lo tanto, desde el enfoque del ciclo económico o del desarrollo económico, la relación se ve caracterizada por tres tipos de problemas: 1) surge una relación directa que tiene una difusa causalidad; 2) se desconocen los móviles individuales que incentivan el crimen; 3 ) toda vez que los estudios son en esta información precarios y de análisis parcial, las conclusiones son acertadas en términos generales, pero sesgadas en lo que respecta a casos particulares.

De modo que, en términos agregados, el comportamiento del crimen, desde la perspectiva teórica, se debe en gran medida a la relación multidimensional que interactúa dinámica y particularmente en un territorio. En otras palabras, propone que las convicciones y motivaciones individuales que incentivan el crimen se deben a cuestiones normativas, estructurales y contextuales. Factores que influyen en la población, al punto de fijar el volumen de actos criminales que se cometen en el territorio durante un periodo de tiempo específico, concepto que, a partir de ahora, va a ser denominado "oferta criminal", tal y como los autores Bandrés y Díez-Ticio (2001) lo definen en su obra.

\section{Metodología}

Esta investigación hace uso del método de indagación cuantitativo, el cual, teniendo en cuenta los planteamientos de los autores referenciados en el apartado anterior, busca examinar las afectaciones que presentan las variables agregadas en la determinación del crimen desde la interacción sistemática de variables sociodemográficas, económicas, institucionales y geográficas ${ }^{5}$. Dado lo anterior, si bien hay enfoques existentes que aprueban la inclusión o exclusión de las variables, al tenerse en cuenta la concepción teórica y de enfoque objetivo que fundamenta la explicación del crimen adoptada en este estudio, hacen que su aplicación se acote únicamente a variables que puedan ser medidas de manera objetiva, y que su sesgo se deba en torno a desviaciones meramente estadísticas. Por lo cual, el método investigativo que se propone no contempla variantes que se relacionen a los componentes propios de la percepción o sus derivados.

\subsection{Desarrollo del modelo matemático y econométrico}

A continuación, se presenta el modelo matemático planteado por la teoría de referencia, esto con el fin de adecuarlo al fenómeno que pretende explicarse en esta investigación. Inicialmente, teniendo en cuenta la descripción matemática preliminar propuesta por Becker (1968) y ampliada por trabajos como los de Núñez et al. (2003),

5 El modelo trabajado deja por fuera efectos de relaciones funcionales propias de las vecindades territoriales y trayectorias urbano - rurales que bien pueden ser objeto de un análisis espacial distinto. Si bien lo espacial no era el foco de la investigación, el escrito aborda tangencialmente el aspecto geográfico que puede ser insumo de otras aplicaciones propias de la econometría espacial. 
Sociedad y Economía N`43 (Mayo - Ago 2021) / e-ISSN: 2389-9050 / e 1029738

Hector Daniel Martínez-Duarte, Angel David Ramírez-Romero y Eder Alexander Botello-Sánchez

Rodríguez (2003), Bandrés y Díez-Ticio (2001) y Martínez (2001), se puede describir al crimen en la Ecuación 1.

$S_{i t}^{D}=f\left(E P_{i t}, E J_{i t}, D O T_{i t}, Y I_{i t}, Y i_{i t}, \mu\right)$

Donde:

$S_{i t}^{D} \quad$ Número de delitos (Oferta agregada del crimen).

$E P_{i t}$ Eficiencia policial.

$E J_{i t} \quad$ Eficiencia de la justicia.

$D O T_{i t}$ Número de agentes de policía.

$Y I_{i t} \quad$ Ingreso legal esperado corriente.

$Y_{i t} \quad$ Ingreso ilegal esperado corriente.

$\mu \quad$ Es un vector sistemático que contiene variables sociodemográficas, económicas, geográficas e institucionales.

Asimismo, la inclusión de las variables de Eficiencia policial, Eficiencia de la justicia y Número de agentes de policía son incluidas en la ecuación principal, debido a que surgen como medidas disuasorias, propias del modelo de ecuaciones simultáneas desarrollado por Núñez et al. (2003); en donde afirma que las mismas se presentan en términos de proporción definidas. Con base en lo anterior, la especificación definida de la variable de Eficiencia policial permite denotar una función de producción que recurra al uso de inputs policiales con outputs probabilísticos de captura y sanción (variable de disuasión). Por lo cual, de acuerdo con el planteamiento de Nagin (1978), se supone la existencia de una Ecuación 2.

$E P_{i t}: f\left(S_{i t}^{D}, D O T_{i t}, Y\right)$

Como producto de la especificación de la Ecuación 2, se puede concluir que del análisis compuesto entre la Ecuación 3 y la Ecuación 4 se hace evidente la presencia de interrelaciones discretas entre los vectores sistemáticos dependientes (endogeneidad). Esto debido a que la formulación matemá- tica especifica, de manera explícita, una formulación con efecto congestión. Núñez et al. (2003) indica que la modelación de los determinantes socioeconómicos de la criminalidad debe considerar en forma explícita la existencia de relaciones endógenas entre la oferta criminal y las características del judicial y policial del país.

En adición a estos planteamientos, autores como Loftin y McDowall (1982), Sandelin y Skogh (1986) y Benson y Cullen (1998), indican los supuestos sistemáticos en el comportamiento de la dotación policial en términos de personal, en donde asumen que existe una relación entre insumos policiales y criminalidad, lo que sustenta la Ecuación 3.

$D O T_{i t}=f\left(S_{i t}^{D}, \mu, E P_{i t}\right)$

Por lo cual, el sistema de ecuaciones que pretenden ser estimadas en la presente investigación viene definido por la unión de la Ecuación 1, 2 y 3; de modo que la Ecuación $4^{6}$ :

$\left\{\begin{array}{l}S_{i t}^{D}=f\left(E P_{i t}, E J_{i t}, D O T_{i t}, Y I_{i t}, Y i_{i t}, \mu\right) \\ E P_{i t}=f\left(S_{i t}^{D}, D O T_{i t}, Y\right) \\ D O T_{i t}=f\left(S_{i t}^{D}, \mu, E P_{i t}\right)\end{array}\right.$

En donde el sistema se estima mediante el modelo de regresión de ecuaciones simultáneas de Máxima Verosimilitud con Información Completa (MVIC). Adicionalmente, con el fin de obtener información que relacione la oferta criminal con la Ecuación 4 en relación de elasticidades, se tiene que el modelo a estimar es presentado en la Ecuación 5.

6 El sistema de ecuaciones cumple con la restricción de exclusión de acuerdo con lo planteado por Wooldridge, J. M. (2010). En el siguiente enlace, se exponen los anexos del modelo, apéndice $B$ y C: https://drive.google.com/file/d/1 Yf5JwT8Li xtzCpyylFivtk3FCv0ZG6DC/view?usp=sharing 


$$
\begin{aligned}
& {[1] \operatorname{Ln} S_{i t}^{D}=\alpha_{1 i t}+b_{11} \ln E P_{i t}+b_{12} \ln E J_{i t}+b_{13} \ln D O T_{i t}+b_{14} Y I_{i t}+b_{15} \ln Y i_{i t}+\mu_{i t}+\tau_{i t}} \\
& {[2] \operatorname{Ln} E P_{i t}=\alpha_{2}+b_{21} \ln S_{i t}^{D}+b_{23} \ln D O T_{i t}+b_{24} \ln Y_{i t}+\tau_{2}} \\
& {[3] \ln D O T_{i t}=\alpha_{3}+b_{31} \ln E P_{i t}+b_{23} \ln S_{i t}^{D}+b_{24}+\ln \text { Área }+\tau_{3}}
\end{aligned}
$$

Donde:

$$
\begin{aligned}
& \mu_{i t}=b_{16} \text { Desemp }_{i t}+b_{17} \text { In } \text { Gini }_{i t}+b_{18} \text { In Cobed }+b_{19} \text { Frontera }+\ldots \\
& \ldots=b_{110} \text { Ln Dens }_{i t}+b_{111} \text { Ln Masc }_{i t}+b_{112} \text { Rural }_{i t}+b_{113} \text { Ln Etar }_{i t}+\ldots+ \\
& \ldots=b_{114} \text { Region }_{i t}+b_{115} \text { Ln Pobmon }_{i t}
\end{aligned}
$$

$\tau_{j_{i t}}:$ El término de la ecuación j analizada.

Adicionalmente, la muestra se compone por los 32 departamentos del país más el distrito capital de Bogotá para los 8 años. La tarea de los autores fue analizar los crímenes en Colombia entendidos como: delitos sexuales, hurtos y homicidios. Para el análisis, se utilizaron los datos del Observatorio del Delito de la Policía Nacional (ODPN). Asimismo, se recurrió a datos del Banco de la República (BanRep), el Departamento Nacional de Estadística y el Departamento Nacional de Planeación colombiano. En la Tabla A1 del Anexo, se explican ampliamente las variables que comprende el modelo.

Por otra parte, las variables abordadas en este estudio responden a cuatro dimensiones, las cuales, desde los diversos planteamientos abordados, aportan al comportamiento agregado del crimen. Por tal motivo, la Tabla 1 clasifica y organiza cada una de las variables de acuerdo con la dimensión a la que pertenece.

\subsection{Hipótesis}

Debido a que en el sistema de ecuaciones formulado se presentan algunas variables de forma endógena, se hace necesario resumir los resultados esperados a priori de cualquier estimación, como se expone en la Tabla 2. En ese orden de ideas, cabe resaltar que algunas de las variables mostradas en este modelo econométrico aparecen en más de una ecuación, razón por la cual, se sugieren indicios de peso teórico que señalan la presencia de endogeneidad por efecto congestión en el planteamiento especificado.

\section{Resultados}

Dada la forma funcional presentada anteriormente, el modelo que ha sido producto de esta investigación se presenta en la Tabla 3 , la cual resume los principales resultados obtenidos al momento de realizar la estimación.

\begin{tabular}{|c|c|c|c|c|c|c|c|}
\hline \multicolumn{8}{|c|}{ Tabla 1. Clasificación de las variables } \\
\hline \multicolumn{2}{|c|}{ Variables Sociodemográficas } & \multicolumn{2}{|c|}{ Variables Económicas } & \multicolumn{2}{|c|}{$\begin{array}{c}\text { Variables } \\
\text { Instifucionales }\end{array}$} & \multicolumn{2}{|c|}{$\begin{array}{c}\text { Variables } \\
\text { Geográficas }\end{array}$} \\
\hline Tasa de cobertura educativa & TC & Ingreso legal esperado & YI & Eficiencia Judicial & EJ & Frontera & Front \\
\hline Número de crímenes & $S D$ & Ingreso ilegal esperado & Yi & Eficiencia Policial & $E P$ & No rural & Rural \\
\hline Densidad poblacional & Dens & Gini & Gini & Dotación Policial & Dot & Región & Región \\
\hline Masculinidad & Masc & Desempleo & Des & & & Área & Área \\
\hline \multirow[t]{2}{*}{ Etariedad } & Etar & Pobreza & Pobm & & & & \\
\hline & & Crecimiento del PIB & Lny & & & & \\
\hline
\end{tabular}

Asimismo, teniendo en cuenta lo anterior, se muestran las particularidades estadísticas de cada una de las ecuaciones estimadas. La Tabla 4 resume los principales hallazgos obtenidos. 
Sociedad y Economía N 43 (Mayo - Ago 2021) / e-ISSN: 2389-9050 / e 1029738

Hector Daniel Martínez-Duarte, Angel David Ramírez-Romero y Eder Alexander Botello-Sánchez

Tabla 2. Hipótesis y signos esperados en las ecuaciones

Variables Independientes

Variables dependientes

Número de Crímenes Eficiencia Policial

$\begin{array}{cc}+ & + \\ \text { No } & - \\ + & \text { No }\end{array}$

Ef. Policial

Dot. Policial

Eficiencia Judicial

Y legal

$Y$ ilegal

$\operatorname{Ln}(\mathrm{PIB})$

Área

U

Gini

Frt

Den

Masc

Rural

Estar

Cobert

Reg

Pobreza

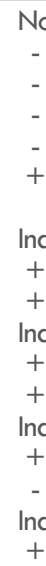

Fuente: elaboración propia con base en las relaciones causales descubiertas por Rodríguez (2003), Bandrés y Díez-Ticio (2001) y Becker (1968).

Tabla 3. Resultados de estimación del sistema de ecuaciones

Método de estimación:

Datos del Sistema

Convergencia
Máxima Verosimilitud con Información Completa

5016 observaciones balanceadas

Lograda tras 206 interacciones.

Covarianza de los coeficientes computada usando resultados del producto de gradientes.

\begin{tabular}{|c|c|c|c|c|}
\hline Variable & Coeficiente & Error estándar & Estadístico Z & Probabilidad \\
\hline Constante Ec. 1 & $-4.798,00$ & $1.340,00$ & $-3,57800$ & 0,00 \\
\hline $\operatorname{Ln}(E \mathrm{E})$ & 0,12 & 0,04 & 3,03500 & 0,00 \\
\hline $\operatorname{Ln}(\mathrm{Ep})$ & $-0,80$ & 0,09 & $-8,88300$ & 0,00 \\
\hline Ln (Dot) & 0,87 & 0,14 & $6,362\} 00$ & 0,00 \\
\hline $\operatorname{Ln}(Y i)$ & 0,18 & 0,06 & 3,24800 & 0,00 \\
\hline $\operatorname{Ln}(Y I)$ & 0,04 & 0,02 & 1,75900 & 0,08 \\
\hline Ln (Desem) & 0,01 & 0,02 & 0,50 & 0,62 \\
\hline Ln (Gini) & 0,13 & 0,08 & 1,55900 & 0,12 \\
\hline Ln (Cobed) & 0,23 & 0,09 & 2,47400 & 0,01 \\
\hline Frontera & 0,00 & 0,02 & $-0,11$ & 0,92 \\
\hline Ln (Dens) & 0,23 & 0,07 & 3,26700 & 0,00 \\
\hline Ln (Masc) & $-0,35$ & 0,44 & $-0,78$ & 0,44 \\
\hline Rural & $-0,02$ & 0,02 & $-0,87$ & 0,39 \\
\hline Ln (Etar) & $-0,06$ & 0,08 & $-0,80$ & 0,43 \\
\hline Región & 0,03 & 0,01 & 2,49100 & 0,01 \\
\hline Ln (Pobmon) & 0,01 & 0,01 & 0,76 & 0,45 \\
\hline Constante Ec. 2 & $2.903,00$ & 0,20 & 1,478800 & 0,00 \\
\hline $\operatorname{Ln}(S D)$ & 0,68 & 0,05 & $1,4377,00$ & 0,00 \\
\hline $\operatorname{Ln}(\mathrm{Dot})$ & $-2.798,00$ & 0,39 & $-7,18700$ & 0,00 \\
\hline $\operatorname{Ln}(Y)$ & $2.383,00$ & 0,39 & $6,109,00$ & 0,00 \\
\hline Constante Ec. 3 & $-1.016,00$ & 0,76 & $-1,34600$ & 0,18 \\
\hline $\operatorname{Ln}(\mathrm{Ep})$ & 0,76 & 0,09 & 8,65900 & 0,00 \\
\hline $\operatorname{Ln}(S D)$ & 0,85 & 0,05 & 1,766300 & 0,00 \\
\hline Ln (Área) & 0,21 & 0,08 & 2,71400 & 0,01 \\
\hline Log. Verosimilitud & $-5.987 .682,00$ & \multicolumn{2}{|c|}{ Criterio de Schwarz } & 41,32200 \\
\hline Log. Verosimilitud Medio & $-6,80200$ & \multicolumn{2}{|c|}{ Criterio de Hannan-Quinn } & 41,12800 \\
\hline Criterio de Akaike & 40,99700 & \multicolumn{2}{|c|}{ Error Cuadrático Medio } & 0,76 \\
\hline
\end{tabular}


Sociedad y Economía N 43 (Mayo - Ago 2021) / e-ISSN: 2389-9050 / e1029738

Incidencia multidimensional de la oferta criminal de Colombia desde un enfoque de ecuaciones simultáneas

Tabla 4. Generalidades de las ecuaciones estimadas

\begin{tabular}{|c|c|c|c|c|}
\hline \multirow[t]{5}{*}{ Ecuación 1} & Observaciones evaluadas & 264 & & \\
\hline & R-cuadrado & 0,947 & Media muestral & 7,642 \\
\hline & R-cuadrado ajustado & 0,924 & Desviación típica muestral & 1,647 \\
\hline & Suma explicada & 0,782 & Suma residual de cuadrados & 151,766 \\
\hline & Estadístico Durbin-Watson & 0,540 & Raíz error cuadrático medio & 0,756 \\
\hline \multirow[t]{5}{*}{ Ecuación 2} & Observaciones evaluadas & 264 & & \\
\hline & R-cuadrado & 0,7628 & Media muestral & 4,687 \\
\hline & R-cuadrado ajustado & 0,7600 & Desviación típica muestral & 0,626 \\
\hline & Suma explicada & 0,306 & Suma residual de cuadrados & 24,466 \\
\hline & Estadístico Durbin-Watson & 0,661 & Raíz error cuadrático medio & 0,304 \\
\hline \multirow[t]{5}{*}{ Ecuación 3} & Observaciones evaluadas & 264 & & \\
\hline & R-cuadrado & 0,767 & Media muestral & 8,982 \\
\hline & R-cuadrado ajustado & 0,764 & Desviación típica muestral & 1,460 \\
\hline & Suma explicada & 0,708 & Suma residual de cuadrados & 130,625 \\
\hline & Estadístico Durbin-Watson & 0,531 & Raíz error cuadrático medio & 0,7034 \\
\hline
\end{tabular}

\subsection{Significancia}

De acuerdo con el test de Wald, el modelo multiecuacional estimado es significativo conjuntamente. Es decir, al 95\% de confianza, cada uno de los coeficientes de las 3 ecuaciones estimadas aportan en conjunto para explicar el comportamiento promedio del número de denuncias, la eficiencia policial y la dotación policial respectivamente. Por otra parte, en términos individuales, la elasticidad parcial de variables como el ingreso legal esperado, la tasa de desempleo, el coeficiente de Gini, el porcentaje de masculinidad, la proporción de la población entre 10 y 24 años, y la pobreza monetaria extrema, resultaron ser no significativos estadísticamente. En otras palabras, los resultados sugieren que, para que se afecte la dinámica criminal, se requiere la interacción simultánea (en conjunto) de estas.

Asimismo, en lo que respecta a variables como Frontera, Rural y el Intercepto de la Ecuación 3 , son variables que no afectan la dinámica autónoma de los departamentos en el país. Lo anterior se sustenta en que, con un nivel de confianza del 95\%, no son estadísticamente significativos. Sin embargo, la significancia individual afirma que, en el $60 \%$ de las observaciones, la ruralidad es una condición disuasora. Finalmente, los demás coeficientes estimados (no mencionados anteriormente) son significativos individualmente a la hora de explicar el comportamiento promedio de la Oferta criminal y la ecuación a la que corresponden.

\subsection{Interpretación}

De acuerdo con la evidencia empírica expuesta y desarrollada a lo largo del presente documento, y teniendo en cuenta el modelo estimado anteriormente, se puede intuir con respecto a los parámetros en cada ecuación.

\subsubsection{Ecuación 1}

Manteniendo todo las demás variables constantes, el valor esperado promedio del número de denuncias en los departamentos de Colombia es $0,115 \%$ mayor cuando el sistema judicial de la región incrementa, en 1\%, su fortaleza en términos de eficacia y eficiencia relativa. Además, manteniendo aislados los efectos de las demás variables, un incremento en una unidad porcentual en la eficiencia relativa del sistema policial produce un decrecimiento en promedio de la oferta criminal del territorio en 0,802 unidades porcentuales. Cuando todo lo demás permanece constante, se tiene que incrementos porcentuales en el número de agentes activos de la policía nacional, en la seccional analizada, inducen a aumentos en el número de crímenes que se efectúan en dicha área.

Por otra parte, incrementos en un $1 \%$ en la tasa de desempleo, el ingreso esperado legal corriente, el índice de Gini, el porcentaje de masculinidad, la proporción de población entre 10 y 24 años, y la pobreza monetaria, no provocan ningún tipo de alteración en el comportamiento promedio de la oferta criminal de los departamentos del país. 
Sociedad y Economía N`43 (Mayo - Ago 2021) / e-ISSN: 2389-9050 / e 1029738

Hector Daniel Martínez-Duarte, Angel David Ramírez-Romero y Eder Alexander Botello-Sánchez

En yuxtaposición, bajo supuesto de Céteris Páribus, aumentos porcentuales en la tasa de cobertura educativa provoca un efecto positivo en la oferta criminal del país de $0,234 \%$, en promedio; adicionalmente, un aumento en la densidad poblacional del departamento en un $1 \%$ estimula que el nivel de crímenes en Colombia aumente en promedio un $0,227 \%$. Finalmente, los departamentos que pertenecen a la región Andina poseen en promedio un nivel autónomo de crímenes 0,02\% más alto.

\subsubsection{Ecuación 2}

En lo que respecta a la eficiencia policial, la especificación econométrica planteada expone que, si se aíslan los efectos de las demás variables, cuando el número de crímenes denunciados se incrementa en un $1 \%$, el incremento marginal promedio de la eficiencia policial es de $0,678 \%$. Asimismo, cuando la producción agregada del departamento se eleva en un $1 \%$, entonces el nivel de eficiencia policial se incrementa en $2,383 \%$. Finalmente, en relación con el número de agentes en la región, la evidencia empírica arroja que, tras incrementos porcentuales en el número de agentes, la eficiencia policial se ve disminuida en $2,798 \%$.

\subsubsection{Ecuación 3}

Por último, la relación estimada para el número de agentes expone que, si la eficiencia policial relativa del departamento se incrementa en $1 \%$, entonces el crecimiento de la dotación policial va a elevarse en $0,758 \%$. De igual manera, cuando el número de crímenes se incrementa en una unidad porcentual, el número de agentes activos se incrementa en 0,84\%. Además, cuando el área del departamento evaluado es un $1 \%$ mayor, entonces el número de policías activos dentro del territorio es 0,209\% veces mayor.

\subsection{Análisis de ajuste del modelo}

Tras efectuar el modelo econométrico en la Ecuación 1, se pudo identificar un alto nivel de ajuste entre las observaciones y las estimaciones, en donde el comportamiento del crimen en Colombia explicado por el vector sistemático tiene una distribución gaussiana más marcada que la variable efectiva (ver histogramas al costado izquierdo). En resumen, la Figura 1 evidencia la existencia de una alta determinación entre la variable sintética y la observada $(94,7 \%)$, esto como resultado de la simultánea

Figura 1. Comparación gráfica entre número de crímenes predichos y número de crímenes efectivos en Colombia (2010 a 2017)

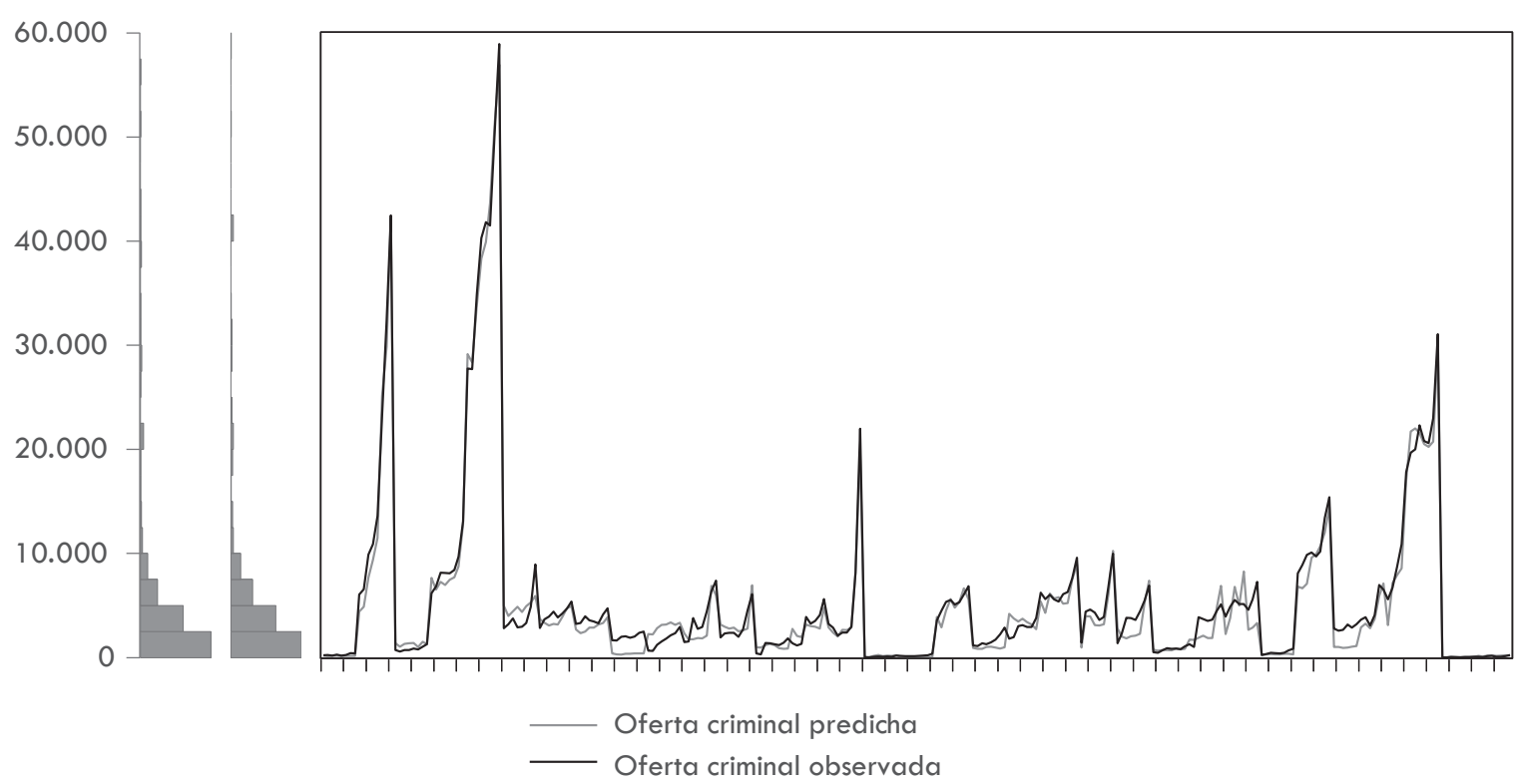

Fuente: elaboración propia. 
interacción entre los determinantes sociodemográficos económicos, geográficos e institucionales.

En la Figura 2, la aplicación del modelo expone que las predicciones derivadas de la especificación presentada en la Ecuación 2 reflejan una relación cercana con el índice de relación entre inputs policiales y resultados operativos construido. Lo cual corrobora una dependencia entre la eficiencia policial y los recursos destinados a la mitigación del crimen, en donde las variaciones porcentuales de estas dos variables, en conjunto, se ajustan al $76,26 \%$ de los datos y, de manera simultánea, la ecuación estimada se corresponde, recíprocamente, con el $87,4 \%$ de los departamentos del país durante los ocho años.

Finalmente, en cuanto a la Figura 3 , se puede ver la comparativa entre el capital humano de la Policía Nacional de Colombia; una brecha leve entre las dos variables analizadas, en donde la predicción desarrollada por la estimación de los parámetros de la Ecuación 3 presenta sobreestimaciones significativas en el $13,47 \%$ de los de- partamentos analizados. Asimismo, los resultados muestran que la serie predicha presenta una menor distribución normal, lo que expone un comportamiento de la dotación policial sectorizada acorde al territorio, en donde el área y la participación económica juegan un papel importante (ver histogramas al costado izquierdo).

\section{Conclusiones}

En lo que respecta al referente teórico de esta investigación, se puede concluir que, si bien el planteamiento de Becker (1968) descansa en el supuesto de racionalidad del individuo y pese a que este argumento puede ser altamente cuestionado por las nuevas escuelas de pensamiento económico, la abstracción de la sociedad que se deriva de esta simplificación, permite comprender sustancialmente el fenómeno criminal de manera aplicada. Esto a un costo relativamente bajo si se tienen en cuenta los resultados agregados del modelo. Además, el enfoque psíquico, individual y determinista que adopta el modelo Beckeriano, logra englobar las dimensiones delictivas al punto de identificar las causas que pueden transformar

Figura 2. Comparación gráfica entre Eficiencia policial predicha y

Eficiencia policial efectiva en Colombia (2010 a 2017)

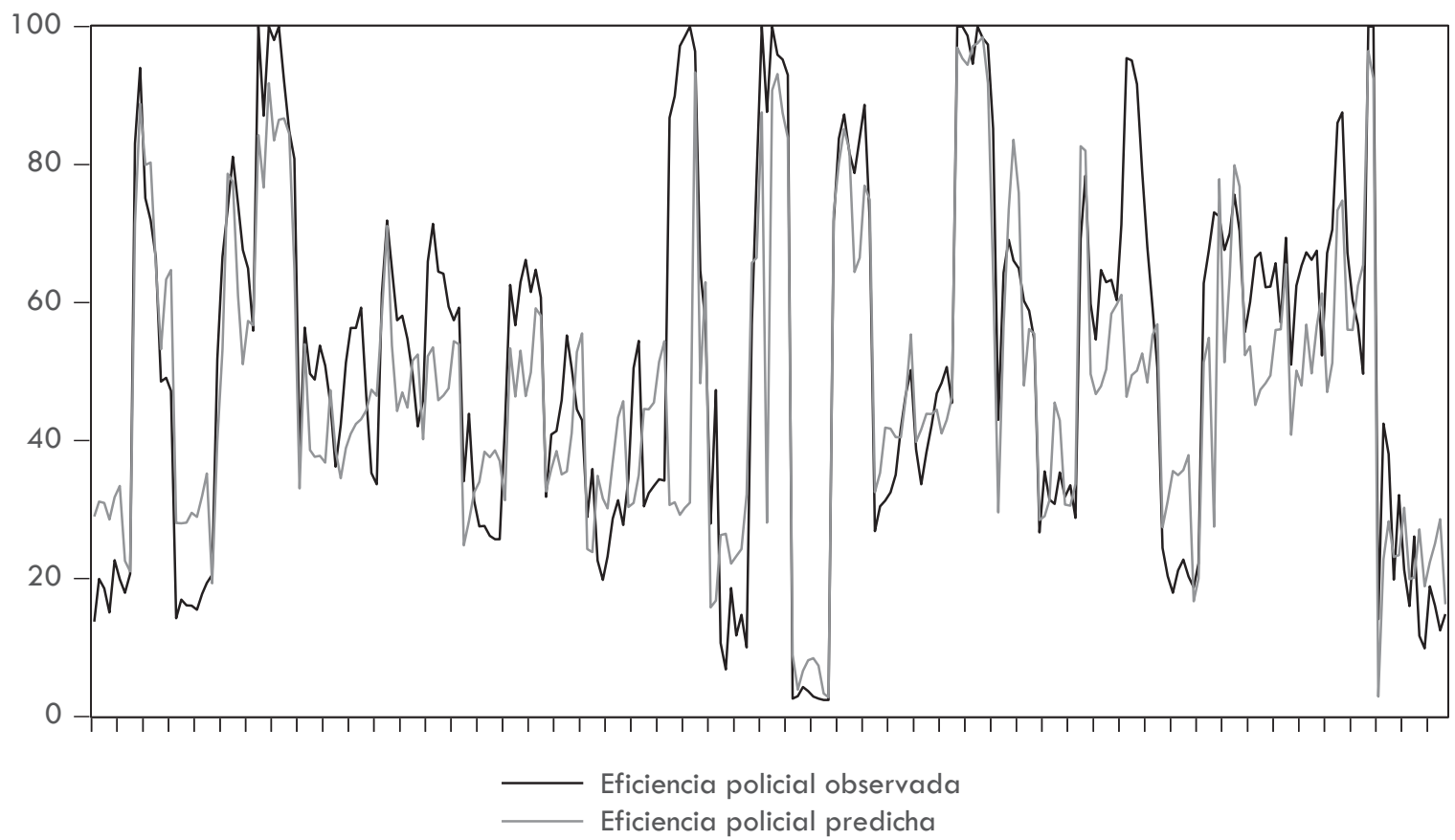

Fuente: elaboración propia. 
Sociedad y Economía N`43 (Mayo - Ago 2021) / e-ISSN: 2389-9050 / e 1029738

Hector Daniel Martínez-Duarte, Angel David Ramírez-Romero y Eder Alexander Botello-Sánchez

Figura 3. Comparación gráfica entre Dotación policial predicha y Dotación policial efectiva en Colombia (2010 a 2017)

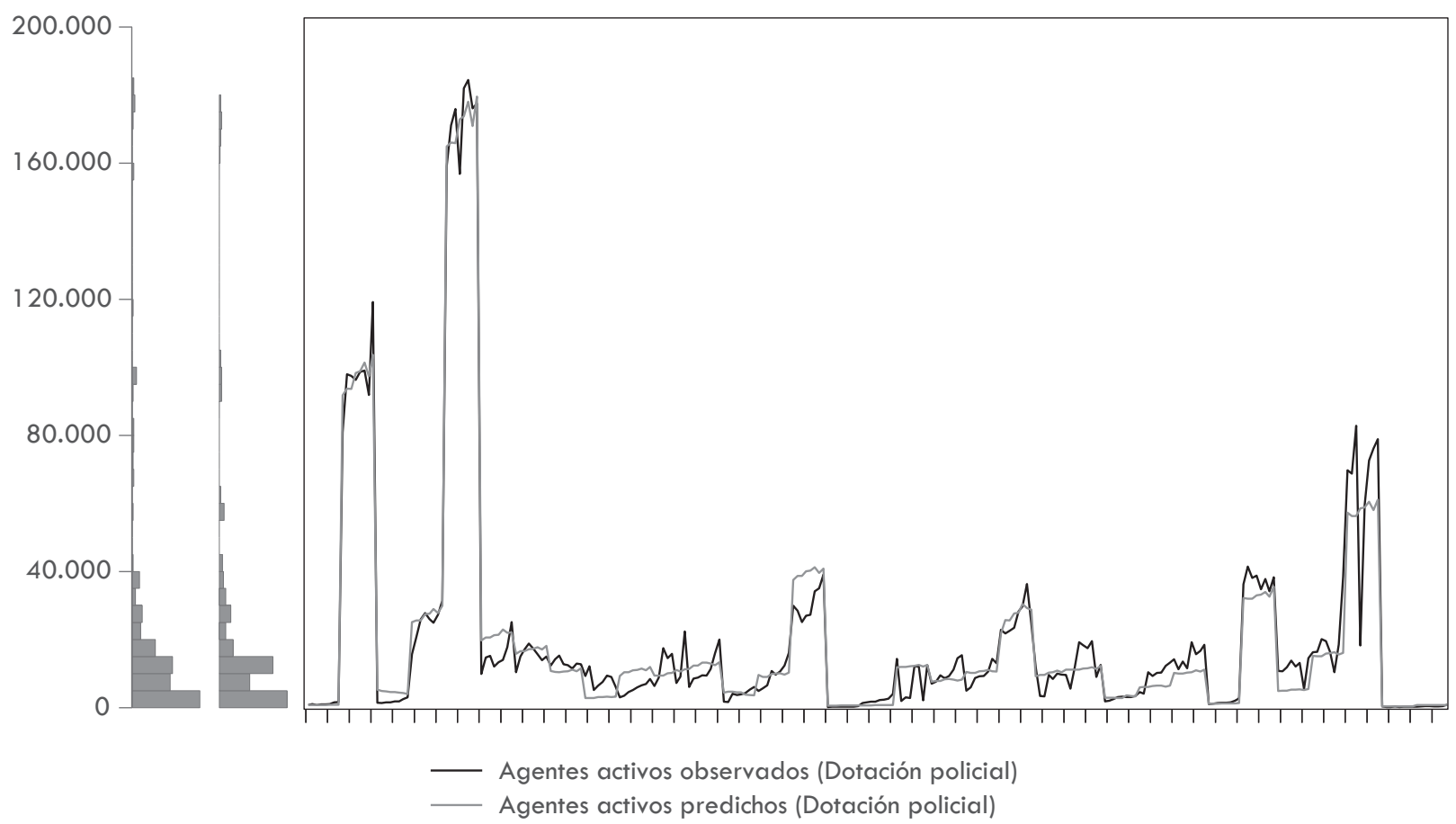

Fuente: elaboración propia.

y desplazar la oferta criminal. Posteriormente, el modelo Beckeriano, ampliado y adoptado en este estudio, propuso que el nivel de crímenes mínimo posible se halla cuando el costo marginal -derivado de la eficacia policial- se iguala al producto entre el castigo monetario recibido por criminal y la reducción del crimen derivado de un aumento en la probabilidad de captura.

A la hora de hablar de homicidios, en el transcurso del periodo analizado se puede vislumbrar una tendencia a la baja desde 2012; por otra parte, el nivel de denuncias por hurtos ha aumentado desde 2010, lo cual sugiere que el crimen en el país podría estar sufriendo una metamorfosis en cuanto a desenvolvimiento y actividades económicamente factibles. Respecto a las hipótesis planteadas a priori, se puede concluir que se cumplen en su mayoría los signos trazados por la teoría de referencia, esto exceptuando el caso de la Eficiencia judicial, la Dotación policial, el Ingreso legal esperado corriente, la Cobertura educativa, la Densidad poblacional, la Etariedad, (en el caso de la Ecuación 1), la Dotación policial (en el caso de la Ecuación 2), la Eficiencia policial (en la Ecuación 3).

Finalmente, en lo que respecta a las conclusiones que se desglosan de la estimación del modelo, se puede señalar que en cada una de las dimensiones analizadas hay al menos dos variables que ayudan a la disuasión del crimen. Por lo cual, la incidencia de los factores sociodemográficos, económicos, institucionales y geográficos en la oferta del crimen proveen una explicación objetiva que argumenta la existencia del nexo entre las dimensiones y el fenómeno criminal.

En torno a las instituciones, la eficacia en la administración y empleo de los recursos físicos del sector público son una opción que permite reprimir el impacto de la oferta del crimen, puesto que la misma reduce la magnitud del crimen 2,31 veces más efectivamente que las demás. En cuanto a lo geográfico, se tiene que la ruralidad es una condición que provee 15 veces menos crímenes que las secciones con territorio fronterizo; también el efecto combinatorio proporcional del número 
de crímenes y la eficiencia policial de la seccional departamental provocan un efecto considerablemente elástico en la vinculación de personal a la Policía Nacional. Los incrementos porcentuales en la masculinidad, etariedad y pobreza monetaria alteran la dinámica criminal en $-0,34 \%,-0,06 \%$ y $0,009 \%$, respectivamente, en 1 de cada 2 departamentos aproximadamente.

Este modelo es una mejor aproximación puesto que, al tener en cuenta las relaciones endógenas, expresa resultados más verosímiles a la aplicación a lo largo del territorio, en la medida en que disipa la heterogeneidad en las divisiones administrativas. Por lo tanto, el promedio es más representativo en cada uno de los casos. Finalmente, del trabajo de investigación aquí desarrollado se puede concluir que la oferta criminal del país, durante el periodo comprendido entre 2010 y 2017 , abarca un concepto multicausal e interdisciplinario, por lo cual, se vio influenciada por diversos factores sociodemográficos, económicos, institucionales y geográficos, los cuales interactuaron en la fijación del crimen de manera consistente.

\section{Anexo}

\section{Tabla A1. Definición de las variables}

Oferta Criminal $\left(S_{i t}^{D}\right)$ : $\quad$ La oferta criminal puede ser definida como una analogía de la oferta laboral, en la cual los seres humanos, en vez de ofrecer su mano de obra para el proceso productivo, ofrecen sus capacidades para las actividades delictivas. Por lo cual, la cantidad de crímenes que se disponen en la economía en determinado momento no es otra cosa más que una de las fuerzas del mercado delictivo de dicho territorio. Con base en lo anterior y sabiendo de antemano que las limitaciones en la información imposibilitan el acceso pleno a los datos en el país, se recurre a emplear el número de delitos denunciados que calcula el ODPN. Dicha variable se adopta como proxy, debido a que acoge la esencia del fenómeno criminal en cuestión. Puesto que la serie es otorgada de manera individual, se describe como:

$\mathrm{S}_{\text {it }}^{\mathrm{D}}=$ Delitos Sexuales $_{\text {it }}+$ Homicidios $_{\text {it }}+$ Hurtos $_{\text {it }}$ Donde: i y $t$ representan indicativos para las secciones y el periodo.

Índice de Eficiencia Policial (EP. $)$ : $\quad$ Esta variable representa la tasa en la cual la policía efectúa la imputación de cargos a los actores criminales en relación con la cantidad de insumos. La inclusión de esta variable surge como factor disuasor que contrae el comportamiento agregado del crimen. La variable se define de la forma:

$E P_{i f}=$ Capturas $_{i f} /$ Denuncias $_{i t} * \theta i t$

Donde:

i y $t$ representan indicativos para las secciones y el periodo, respectivamente.

$\theta$ hace alusión a un número índice de programación lineal" que calcula la eficiencia relativa de la unidad medida** . La variable comprende el rango de desenvolvimiento acotado de 0 a 1 , en donde 1 representa eficiencia relativa total y 0 la ausencia del mismo concepto.

\begin{aligned} \hline Dotación de número de oficiales (DOT): & La variable expone el número de agentes activos en la institución colombiana \\ & dedicada a la disuasión del crimen desde el ámbito operativo. Por su parte, esta \\ & variable se presenta en unidades y no distingue entre cargo o posición jerárquica que \\ & ocupan los individuos dentro del organismo de control civil. \end{aligned}

Ingreso esperado ilegal corriente $\left(Y_{L_{t}}\right)$ : La variable PIB per-cápita de la región i en el periodo $\dagger$ cumple con los requerimientos necesarios para compilar el efecto que desea evaluarse en este estudio. Asimismo, teniendo en cuenta que el modelo Beckeriano se despoja del análisis racional de expectativas de largo plazo, la variable se expresa en pesos corrientes.

Eficiencia Judicial $\left(E J_{i t}\right)$ :

Esta variable hace alusión al desempeño de la entidad encargada en Colombia del seguimiento y acompañamiento del proceso jurisprudencial de los agentes delictivos, por lo cual, se define de la forma:

$E J_{i t}=$ Judicialización $_{i t} /$ Capturas $_{i t} * \vartheta_{i t}$

Nota: * La estimación de tipo no paramétrica que se efectúa para el cálculo de la variable Theta se presenta en el apéndice $A$ el enlace: https://drive.google.com/file/d/1Yf5JwT8LixtzCpyylFivtk3FCvOZG6DC/view?usp=sharing ** Unidad de medida define, en este caso particular, al departamento i en el año $\dagger$ analizado. Se denomina bajo este nombre puesto que hace alusión a su traducción literal del inglés (Decision-Making Unit-DMU). 
Sociedad y Economía N 43 (Mayo - Ago 2021) / e-ISSN: 2389-9050 / e1029738

Hector Daniel Martínez-Duarte, Angel David Ramírez-Romero y Eder Alexander Botello-Sánchez

Tabla A1. Definición de las variables (continuación)

Desempleo $\left(U_{i j}\right)$ : $\quad$ Esta variable está expresada en porcentaje y hace alusión a la proporción de la población económicamente activa del departamento i en el periodo † que no es absorbida por el mercado laboral local.

Desigualdad (Gini $i_{i t}$ :

Si bien la desigualdad puede representar un concepto amplio y de tipo descriptivo, esta variable se incluye en el modelo para representar el nivel de concentración de la riqueza en relación con el crimen; la misma se encuentra expresada en número índice.

Pobreza Monetaria $\left(\right.$ Pobmon $\left._{i t}\right)$ :

La Pobreza Monetaria es la variable que indica el porcentaje de personas en el periodo que tienen "un ingreso per cápita del hogar por debajo de la línea de pobreza según el dominio geográfico" (DANE, 2020, p. 5).

Frontera (Front $t_{i t}$ : $\quad$ Sea definida como una variable binaria que toma el valor de uno (1) cuando la sección analizada (Departamento) tiene una ubicación territorial que colinda con territorios aledaños al país; y valor de cero (0) en otro caso.

Densidad poblacional ( Densidad $_{i t}$ ): $\quad$ Considérese esta variable como el nivel de saturación de población en el territorio evaluado. La variable se basa en el número de habitantes por cada mil kilómetros cuadrados evaluados $\left(\mathrm{Km}^{2}\right)$.

Masculinidad $\left(\right.$ Masc $\left._{i f}\right)$ : $\quad$ Esta variable representa la proporción de población en la sección evaluada que es hombre. Núñez et al. (2003) plantea que la oferta criminal presenta inclinaciones demográficas de diversa índole, en especial las relacionadas con el género. La variable se muestra como porcentaje de hombres en el departamento frente al número de habitantes.

Ruralidad (Rural ${ }_{i j}$ ) Esta variable dicotómica toma el valor de 1 cuando el departamento analizado tiene un nivel de población por encima de la mediana y toma el valor de 0 en otro caso ${ }^{* * *}$.

Ingreso Esperado Legal $\left(Y_{\text {lit }}\right)$

Esta variable representa el valor esperado promedio que reciben los individuos del territorio. Si bien existen autores que la denotan como la desviación porcentual entre la producción local con la nacional, el Departamento Administrativo Nacional de Estadística (DANE) define la unidad de gasto como "la persona que atiende sus propios gastos, o al grupo de personas que comparten la vivienda y tienen arreglos para satisfacer en común sus necesidades esenciales" (DANE, 2009, citado en DNP, 2012, p. 7).

Región $\left(\right.$ Region $\left._{i \dagger}\right)$ :

Esta variable dicotómica toma el valor de 1 cuando el departamento analizado pertenece a la región Andina y toma el valor de 0 en otro caso*****

Etariedad $\left(\right.$ Etar $\left._{i t}\right)$ :

Esta variable indica la proporción de la población en el territorio evaluado perteneciente al rango 10-24 años. La variable no es multiplicada por cien, de modo que se expresa en forma de escalar.

Tasa de Cobertura Educativa Neta (TC.): Esta variable indica la proporción de la población en edad de estudiar (hasta los 18 años) que se encuentran matriculados en instituciones de educación básica oficial y no oficial en relación con la población total en edad de estudiar del departamento; la variable no es multiplicada por cien, de modo que se expresa en forma de escalar.

Crecimiento del PIB $\operatorname{Ln}\left(Y_{i t}\right)$ :

Esta variable expone los cambios porcentuales de la economía del departamento administrativo $t$ en el año $i$. La variable se expresa como el logaritmo natural del producto interno bruto departamental.

Nota: *** Aunque Núñez et al. (2003) evalúa la densidad demográfica del territorio teniendo en cuenta poblaciones superiores a 100.000 habitantes, esta valoración funciona a nivel municipal mas no a nivel departamental. Con el fin de diversificar los resultados de la variable exógena, se recurre a aplicar una medición diferente. **** Se elige esta región por dos razones: 1 ) el $64 \%$ de las 23 principales ciudades se ubican en dicha región; 2) los niveles de institucionalidad en la región Andina son predominantes en lo que respecta a las demás.

Fuente: elaboración propia.

\section{Referencias}

Anzola, M. G., Iglesias, C., Petrucci, A. R. y Prado-Álvarez, G. (2005). Delincuencia juvenil en Paraná y su relación con las condiciones de exclusión social. Ciencia, docencia y tecnología, 16(31), 49-94.

Balbo, M. y Posadas, J. (1998). Una primera aproximación al crimen en la Argentina (Documentos de Trabajo $\mathrm{n}^{\circ}$ 10). UNLP. http://sedici.unlp.edu.ar/handle/10915/3513

Bandrés, E. y Díez-Ticio, A. (2001). Delincuencia y acción policial, Un enfoque económico. Revista de Economía Aplicada, 9(27), 5-33. 
Sociedad y Economía N 43 (Mayo - Ago 2021) / e-ISSN: 2389-9050 / e1029738

Incidencia multidimensional de la oferta criminal de Colombia desde un enfoque de ecuaciones simultáneas

BBC. (2016). Cuáles son los 6 países de América Latina que están entre los 13 con peores índices de criminalidad en el mundo. https://www.bbc.com/mundo/noticias-america-latina-38171437

Beccaria, C. (1828). Tratado de los delitos y de las penas. En casa de Rosa.

Becker G. S. (1968). Crime and Punishment: An Economic Approach. En N. G. Fielding, A. Clarke y R. Witt (Eds.), The Economic Dimensions of Crime (pp. 13-68). Palgrave Macmillan. https://doi.org/10.1007/9781-349-62853-7_2

Becker, G. S., Murphy, K. M. y Tamura, R. (1990). Human capital, fertility, and economic growth. Journal of political economy, 98(5, Part 2), 323-349. https://doi.org/10.1086/261723

Benson, M. L. y Cullen, F. T. (1998). Combating corporate crime: Local prosecutors at work. UPNE.

Bentham, J. (1789). An introduction to the principles of morals. Athlone.

Buonanno, P. (2003). The socioeconomic determinants of crime. A review of the literature (Working Paper $n^{\circ}$ 63). Università di Milano - Bicocca. https://boa.unimib.it/retrieve/handle/10281/22981/29439/The_ Socioeconomic_Determinants_of_Crime.pdf

Carranza-Romero, J. E., Dueñas-Herrera, X. y González-Espitia, C. G. (2011). An empirical analysis of the relationship between murders and economic activity in Colombia. Estudios Gerenciales, 27(119), 59-77. https://doi.org/10.1016/S0123-5923(11)70157-7

Chabat, J. (2010). El Estado y el crimen organizado trasnacional. Revista de Historia Internacional, 11 (42), 3-14. http://www.istor.cide.edu/archivos/num_42/dossier1.pdf

Collazos, D., García, E., Mejía, D., Ortega, D. y Tobón, S. (2020). Hot spots policing in a high-crime environment: An experimental evaluation in Medellin. Journal of Experimental Criminology, 16, 1-34. https://doi.org/10.1007/s11292-019-09390-1

DANE -Departamento Administrativo Nacional de Estadística-. (2020). Pobreza monetaria en Colombia. Año 2019. https://www.dane.gov.co/files/investigaciones/condiciones_vida/pobreza/2019/Boletin-pobrezamonetaria_2019.pdf

DNP -Departamento Nacional de Planeación-. (2012). Metodologías oficiales y arreglos institucionales para la medición de la pobreza en Colombia. https://colaboracion.dnp.gov.co/CDT/Conpes/Social/150.pdf

Drake, L. M. y Simper, R. (2005). Police efficiency in offences cleared: An analysis of English "Basic command units". International Review of Law and Economics, 25(2), 186-208. https://doi.org/10.1016/j. irle.2005.06.003

Ehrlich, I. (1996). Crime, Punishment, and the Market for Offenses. Journal of economic perspectives, 10(1), 43-67. https://doi.org/10.1257/jep.10.1.43

Estepa, R. I. (2005). El crimen como objeto de investigación histórica. Ohm: Obradoiro de Historia Moderna, (14), 297-318. https://doi.org/10.15304/ohm.14.544

Galindo, L. M. y Catalán, H. (2007). Las actividades delictivas en el Distrito Federal. Revista Mexicana de Sociología, 69(3), 457-484. http://dx.doi.org/10.22201/iis.01882503p.2007.003.6102

Garoupa, N. (2003). Behavioral economic analysis of crime: A critical review. European Journal of Law and Economics, 15(1), 5-15. https://doi.org/10.1023/A:1021152523137

Gómez, M. M. y Serrano, N. G. (2015). La reforma de la Ley de Enjuiciamiento Criminal en 2015. Ediciones Jurídicas Castillo de Luna.

Gómez, S., Mejía, D. y Tobón, S. (2020). The Deterrent Effect of Surveillance Cameras on Crime. (Documentos de trabajo $n^{\circ}$ 20-12). EAFIT. https://repository.eafit.edu.co/bitstream/handle/10784/15941/WP-202012-Santiago\%20Tob\%C3\%B3n.pdf?sequence=2\&isAllowed= 
Sociedad y Economía N`43 (Mayo - Ago 2021) / e-ISSN: 2389-9050 / e 1029738

Hector Daniel Martínez-Duarte, Angel David Ramírez-Romero y Eder Alexander Botello-Sánchez

Loftin, C. y McDowall, D. (1982). Police, crime, and economic theory: An assessment. American Sociological Review, 47(3), 393-401. http://dx.doi.org/10.3886/ICPSR08509

Mancera, A. (2010). Factores socioeconómicos y demográficos de distintas categorías de delitos en Colombia: Prueba desde un panel de datos de las regiones de Colombia. Revista de Economía del Caribe, (2), 202231. http://rcientificas.uninorte.edu.co/index.php/economia/article/viewFile/556/297

Martínez, A. (2001). Economía, crimen y conflicto. Universidad Nacional de Colombia.

Montesquieu, C. (1989). Montesquieu: El espíritu de las leyes. Universidad de Cambridge.

Nagin, D. (1978). Crime rates, sanction levels, and constraints on prison population. Law and Society Review, 12(3), 341-366. https://doi.org/10.2307/3053284

Núñez, J., Rivera, J., Villavicencio, X. y Molina, O. (2003). Determinantes socioeconómicos y demográficos del crimen en Chile. Estudios de economía, 30(1), 55-85.

Ramírez-de-Garay, L. D. (2014). Crimen y economía: una revisión crítica de las explicaciones económicas del crimen. Argumentos, 27(74), 261-290.

Robinson, J. y Urrutia, M. (2007). Economía Colombiana del siglo XX: Un análisis cuantitativo. Fondo de Cultura Económica.

Rodríguez, A. (2003). Los determinantes socioeconómicos del delito en España. Revista española de investigación criminológica, 1, 1-31. https://doi.org/10.46381/reic.v1i0.5

Sandelin, B. y Skogh, G. (1986). Property crimes and the police: An empirical analysis of Swedish data. The Scandinavian Journal of Economics, 88(3), 547-561. https://doi.org/10.2307/3440385

Siegel, L. J. (2011). Criminology. Cengage Learning. https://n9.cl/ri6o

Wooldridge, J. M. (2010). Econometric analysis of cross section and panel data. MIT press. 